\title{
ON A CLASS OF FINITE-DIMENSIONAL LIE ALGEBRAS
}

\author{
RALPH K. AMAYO
}

\begin{abstract}
Over fields of prime characteristic the centre of the universal enveloping algebra of a finite-dimensional Lie algebra contains a finitely generated polynomial algebra over which the universal envelope is a finitely generated module. This result, which is due to Curtis, is crucial in certain investigations of finitely generated soluble Lie algebras and motivates the introduction of the class Max-cu, which will be called the class of Curtis algebras, consisting of Lie algebras whose universal envelopes have the property described above. It has been an open question whether the class Max-cu consists of finite-dimensional Lie algebras. This paper gives an affirmative answer to the question.
\end{abstract}

1. Notation and preliminary results. We employ the notation and conventions of Amayo and Stewart [1].

If $L$ is a Lie algebra then $U(L)$ denotes its universal enveloping algebra. We denote by $\mathbf{F}$ the class of finite-dimensional Lie algebras;

$L \in \operatorname{Max}$ if every subalgebra is finitely generated;

$L \in \operatorname{Max}-\mathrm{u}$ if $U(L)$ is right noetherian;

$L \in \mathrm{Max}-\mathrm{cu}$ if there exists a finitely generated polynomial subring $R$ of the centre of $U(L)$ and a finite number of elements $u_{1}, \ldots, u_{n}$ in $U(L)$ such that $U(L)=R u_{1}+\cdots+R u_{n}$ (equivalently $U(L)$ is a finitely generated $R$-module).

A denotes the class of abelian Lie algebras. By the results of Amayo and Stewart [2], we have Max-cu $\leqslant$ Max- $u \leqslant M a x$, and $F \leqslant$ Max- $u \leqslant$ Max.

Over fields of characteristic zero, $\mathbf{F} \cap \mathbf{A} \leqslant$ Max-cu and over fields of prime characteristic $\mathbf{F} \leqslant$ Max-cu.

Let $L$ be a Lie algebra defined over a field $\mathbf{k}, \mathbf{K}$ an extension field of $\mathbf{k}, U(L)$ the universal envelope of $L$ and $L_{0}=L \otimes_{\mathbf{k}} \mathbf{K}$, the $\mathbf{K}$-Lie algebra extension of $L$. Then $U\left(L_{0}\right)=U(L) \otimes_{\mathbf{k}} \mathbf{K}$. Thus if $L \in \mathrm{Max}-\mathrm{cu}$ and $R=\mathbf{k}\left[z_{1}, \ldots, z_{m}\right] \subseteq$ centre of $U(L)$ such that $U(L)=R u_{1}+\cdots+R u_{n}$, then $U\left(L_{0}\right)=R_{0} u_{1}+\cdots+R_{0} u_{n}$, where $R_{0}=\mathbf{K}\left[z_{1}, \ldots, z_{n}\right]$, whence $L_{0} \in$ Max-cu as a Lie algebra over $K$. We note also that the class Max-cu is closed under homomorphic images and finite direct sums.

Lie algebras in the class Max-cu will be called Curtis algebras.

The proof of the following lemma may be found in Curtis [4] and Amayo and Stewart [1, pp. 225-232].

LEMMA 1.1. Every irreducible module of a Curtis algebra is finite-dimensional.

Presented to the Society, January 24, 1979 under the title Curtis algebras; received by the editors March 28, 1978 and, in revised form, October 31, 1978.

AMS (MOS) subject classifications (1970). Primary 17B65, 17B15, 16A64. 
LEMMA 1.2. Suppose $L$ is a finite-dimensional nonabelian Lie algebra defined over an algebraically closed field $k$ of characteristic zero and suppose also that if $I$ is any nonzero ideal of $L$ then $L / I$ is abelian. Then the following possibilities hold:

(a) $L$ is simple of dimension at least 3.

(b) $L$ is the 2-dimensional nonabelian Lie algebra.

(c) $L$ is the Heisenberg algebra, with a basis $\left\{x_{i}, y_{i}, z ; i=1, \ldots, m\right\}$, where $\left[x_{i}, y_{j}\right]=\delta_{i j} z$,

$$
\left[x_{i}, x_{j}\right]=\left[y_{i}, y_{j}\right]=\left[x_{i}, z\right]=\left[y_{i}, z\right]=0 \text {. }
$$

Proof. Let $R$ be the solvable radical of $L$. If $0<R<L$, then $L / R$ is semisimple and by hypothesis also abelian, a contradiction. Thus $R=0$ or else $R=L$.

In case $R=0, L$ is semisimple, a direct sum of simple ideals and the hypothesis implies that $L$ must be simple and satisfy condition (a).

If $L$ is 2 -dimensional then as $L$ is by hypothesis nonabelian and condition (b) is satisfied. So assume that $L$ has dimension three or more.

Now suppose that $R=L$ and so $L$ is solvable. As $L$ is nonabelian and $L / I$ is abelian for every nonzero ideal $I$ of $L$, the derived algebra $A=[L, L]$ of $L$ is abelian and the unique minimal ideal of $L$. As $\mathbf{k}$ is algebraically closed $A$ is a direct sum of weight spaces under the adjoint representation of $L$ on $A$ and each weight space is an ideal of $L$. Thus there is a linear functional $\lambda$ defined on $L$ with $a(a d x-\lambda(x))^{n}=0$ for all $a$ in $A$ and $x$ in $L$ with $n=n(x)$ a nonnegative integer. In particular, for any $x \in L$ there exists $b \in A-0$, and $\alpha=\lambda(x) \in \mathbf{k}$ with $[b, x]=\alpha b . A$ is the derived algebra and is abelian, hence for any $c$ in the ideal of $L$ generated by $b,[c, x]=\alpha c$. This ideal must be $A$ and as $x$ was arbitrary we have $[a, x]=\lambda(x) a$, for any $a$ in $A$ and $x$ in $L$. In particular, $\mathbf{k} a$ is an ideal of $L$ for any $a$ in $A$, so that $A=\mathbf{k} a$ for some nonzero $a$. Fix any $x$ in $L$. For $y, z \in L,[x, y]$ and $[x, z]$ are in $A$ and so are linearly dependent, whence $c_{L}(x)=\{w \in L \mid[x, w]=0\}$ is a subalgebra of codimension not exceeding one in $L$. If $[a, x] \neq 0$ for some $x$, then $L=\mathbf{k} a+\mathbf{k} x+c_{L}(x) \cap c_{L}(a)$, whence $I=c_{L}(x) \cap c_{L}(a)$ is an ideal of $L$ and must contain $a$, a contradiction. Thus $[a, x]=0$ for all $x$ in $L$ and $A=\mathbf{k} a$ is the centre of $L$. Let $x_{1} \in L-A$, so that for some $y \in L-A,\left[x_{1}, y\right]=\delta a \neq 0$, with $\delta \in \mathbf{k}$ and let $y_{1}=\delta^{-1} y$. Then $\left[x_{1}, y_{1}\right]=a$ and $L=\mathbf{k} x_{1}+\mathbf{k} y_{1}+c_{L}\left(x_{1}\right) \cap c_{L}\left(y_{1}\right)$. Suppose that $x_{1}, \ldots, x_{n-1}$ and $y_{1}, \ldots, y_{n-1}$ have been defined such that $\left[x_{i}, y_{j}\right]=$ $\delta_{i j} a$

$$
\begin{aligned}
& \delta_{i i}=1, \\
& \delta_{i j}=0 \quad \text { if } i \neq j
\end{aligned}
$$

$\left[x_{i}, x_{j}\right]=\left[y_{i}, y_{j}\right]=0$ and $L=k_{n-1}+c_{L}\left(k_{n-1}\right)$, with $k_{n-1}$ the vector subspace spanned by the $x_{i}$ and $y_{i}$. Pick $x_{n} \in C_{L}\left(k_{n-1}\right)-A$. Then for some $y \in L, 0 \neq$ $\left[x_{n}, y\right] \in A$. As $\left[b, x_{n}\right]=0$ for all $b$ in $k_{n-1}, y_{n}$ can be chosen in $c_{L}\left(k_{n-1}\right)$ with $\left[x_{n}\right.$, $\left.y_{n}\right]=a$. Set $k_{n}=k_{n-1}+\mathbf{k} x_{n}+\mathbf{k} y_{n}$. Then $U=c_{L}\left(k_{n-1}\right)=\mathbf{k} x_{n}+\mathbf{k} y_{n}+c_{U}\left(x_{n}\right) \cap$ $c_{U}\left(y_{n}\right)$ so that $L=k_{n}+c_{L}\left(k_{n}\right)$.

As $L$ is finite-dimensional, the process eventually ends to give $L$ the form described in part (c).

Next we will show that in each of these three cases, $L$ cannot be a Curtis algebra. 
EXAMPLE 1.3. Every simple Lie algebra over an algebraically closed field of characteristic zero has irreducible modules of infinite dimension (cf. [5, Chapter VII, Theorem 3]; choose the highest weight not equal to a dominant integral weight). On the other hand, by Lemma 1.1, if such an algebra is a Curtis algebra, its modules are finite-dimensional. Hence such an algebra cannot be a Curtis algebra.

EXAMPLE 1.4. Let $M$ be the polynomial ring $\mathbf{k}\left[t_{1}, \ldots, t_{m}\right]$ and let the Heisenberg algebra $L=\left\langle x_{1}, \ldots, x_{m} ; y_{1}, \ldots, y_{m} ; z\right\rangle$ act on $M$ by $f x_{i}=t_{i} f, f y_{i}=\partial f / \partial t_{i}$, $f z=f$, for each $f=f\left(t_{1}, \ldots, t_{n}\right) \in M$. Then it is easily checked that $M$ becomes an irreducible $L$-module under this action. As $M$ is infinite-dimensional then by Lemma 1.1, $L$ cannot be a Curtis algebra.

EXAMPLE 1.5. Let $L=\langle x, y\rangle$ with $[x, y]=x$ be the 2-dimensional nonabelian Lie algebra over $\mathbf{k}$ and let $U$ be the universal enveloping algebra of $L$. Then the products $x^{i} y^{j}, i, j \geqslant 0$, form a $\mathbf{K}$-basis for $U$. The relations $y^{r} x=x(y-1)^{r}$ and $y x^{r}=x^{r} y-r x^{r}$ show that the centre of $U$ is $\mathbf{k}$, whence $U$ cannot be a finitely generated module over its centre and so $L$ cannot be a Curtis algebra.

THEOREM 1.6. A finite-dimensional Lie algebra over a field of characteristic zero is a Curtis algebra if and only if it is abelian.

Proof. The implication one way is clear. Conversely let $L$ be a finite-dimensional nonabelian Lie algebra over a field $\mathbf{k}$ of characteristic zero. Suppose if possible that $L$ is a Curtis algebra. Let $\mathbf{K}$ be the algebraic closure of $\mathbf{k}$ and $H=L \otimes_{\mathbf{k}} \mathbf{K}$. Then $H$ is a finite-dimensional nonabelian Curtis algebra over $\mathbf{K}$, and any quotient of $H$ is a Curtis algebra. Let $N=H / I$, where $I$ is an ideal of $H$ maximal with respect to $H / I$ being nonabelian. Then as every quotient of $N$ by a nonzero ideal is abelian it follows that $N$ is either simple, 2-dimensional solvable or the Heisenberg algebra, whence by Examples 1.3 to $1.5, N$ cannot be a Curtis algebra, a contradiction.

2. The infinite-dimensional case. Let $\mathbf{X}$ be the class of Lie algebras $L$, such that $U(L)$ is finitely generated as a module over its centre. Then $\mathbf{A}<\mathbf{X}$ and so $\mathbf{X} \Varangle \mathbf{F}$. Denote by Fin-A the class of Lie algebras whose abelian subalgebras are finite-dimensional and by $\mathbf{N}$ the class of nilpotent Lie algebras. We note that the class $\mathbf{X}$ is closed under finite direct sums, quotients and field extensions.

TheOREM 2.1. $\mathbf{X} \cap$ Fin-A $\leqslant \mathbf{F}$.

Proof. Let $0 \neq L \in \mathbf{X} \cap$ Fin-A and let $A$ be the underlying vector space $L$. Consider $A$ as an $L$-module and so $U$-module under the adjoint action: For $a \in A$ and $x, x_{1}, \ldots, x_{r} \in L, a \cdot 1=a, a \cdot x=[a, x], a \cdot\left(x_{1} x_{2} \ldots x_{r}\right)=(a \cdot$ $\left.\left(x_{1} \ldots x_{r-1}\right)\right) \cdot x_{r}$. Then for each subspace $X$ of $L, A_{1}(X)=\{a \in A: a \cdot x=0$ for all $x \in X\}$ is the subspace of $A$ corresponding to $C_{L}(X)$. Let $U=U(L)=u_{1} Z$ $+\cdots+u_{m} Z$ where $Z$ denotes the centre of $U$. Then for any $u \in U, z$ in $Z$ and $a$ in $A$ we have $(a \cdot z) \cdot(u)=a \cdot(z u)=a \cdot(u z)=(a \cdot u) \cdot z$. Thus $A_{1}(X)$ is a $Z$-submodule of $A$ and hence $A_{1}(X) U=A_{1}(X) u_{1}+\cdots+A_{1}(X) u_{m}$ is the underlying space of some ideal of $L$ (ideal generated by $C_{L}(X)$ ). 
Now let $X$ be a maximal abelian subalgebra of $L$. Then $X \neq 0$ and $X=C_{L}(X)$ and as $L \in$ Fin-A, $X$ is finite-dimensional. Thus $A_{1}(X)$ and so $A_{1}(X) U$ are finite-dimensional. On the other hand, $A_{1}(X) U$ is an ideal of $L$ and the action of $L$ on this ideal induces a Lie homomorphism of $L$ into the finite-dimensional space End $\left[A_{1}(X) U\right]$ whose kernel is $C_{L}\left(A_{1}(X) U\right)$. Thus $L / C_{L}\left(A_{1}(X) U\right)$ is finite-dimensional. Now $X \leqslant A_{1}(X) U$ so that $C_{L}\left(A_{1}(X) U\right) \leqslant C_{L}(X)=X$ and hence $L$ must be finite-dimensional.

For a Lie algebra $L$ the adjoint action of $L$ on itself can be extended to a module action of $L$ on $U(L)$ via

$$
\begin{aligned}
\left(x_{1} \ldots x_{r}\right)(a d x) & =\left[x_{1} \ldots x_{r}, x\right] \\
& =\sum_{i=1}^{r}\left(x_{1} \ldots x_{i-1}\right)\left(\left[x_{r}, x\right]\right)\left(x_{i+1} \ldots x_{r}\right)
\end{aligned}
$$

where $x, x_{1}, \ldots, x_{r} \in L$ and $x_{1} \ldots x_{r} \in U(L)$. Thus if $L$ is nilpotent of class $c$ say then it is easily verified that $\left(x_{1} \ldots x_{r}\right)(a d x)^{r c}=0$. So if $u \in U, z \in Z$ (Z the centre of $U=U(L))$ and $x \in L$ then as $U$ is spanned by products of the form $x_{1} \ldots x_{r}$, we can find $s=s(u, x)$ such that $u(a d x)^{s}=0=\left(u(a d x)^{s}\right) z=$ $(u z)(a d x)^{s}$.

THEOREM 2.2. $\mathbf{X} \cap \mathbf{N}=\mathbf{A}$ over fields of characteristic zero.

Proof. Let $L \in \mathbf{X} \cap \mathbf{N}$ and $U=U(L)=u_{1} Z+\cdots+u_{m} Z$ with $Z$ the centre of $U$. From above we can find for each $x$ in $L$ a positive integer $n=n(x)$ such that for any $z$ in $Z,\left(u_{i} z\right)(a d x)^{n}=0$ for $i=1, \ldots, m$ and hence for any $u$ in $U$, $u(a d x)^{n}=0$ and $n=n(x)$. Suppose, if possible, that $L$ is not abelian. Then we can find $x, y$ in $L$ and $z$ in the centre of $L$ and so in $Z$ such that $z \neq 0$ and $y(a d x)=[y, x]=z$. From this we have for any positive integers $k, r$ with $k \geqslant r$, $y^{k}(a d x)^{r}=\left(\begin{array}{l}k \\ r\end{array}\right)(r !) z^{r} y^{k-r}$. In particular, if $n=n(x)$ as above we have $0=y^{n}(a d x)^{n}$ $=(n !) z^{n} \neq 0$ (field of characteristic zero), a contradiction. Therefore, $L$ must be abelian. So $\mathbf{X} \cap \mathbf{N} \leqslant \mathbf{A} \leqslant \mathbf{X} \cap \mathbf{N}$ and the result is proved.

THEOREM 2.3. If $L$ is a Lie algebra in the class $\mathbf{X}$ over an algebraically closed field then there exists a positive integer $m=m(L)$ such that every finite-dimensional irreducible $L$-module has dimension not exceeding $m$.

Proof. Let $\mathbf{k}$ be the field in question and $U=U(L)=u_{1} Z+\cdots+u_{m} Z$ as before, and let $V$ be a finite-dimensional irreducible $L$-module and so a $U$-module. As the field is algebraically closed it follows by Schur's lemma (or one can show this directly) that the centre $Z$ of $U(L)$ acts by scalars on $V$. Hence, if $0 \neq v \in V$, then $V=v U=\sum_{i=1}^{m} v u_{i} Z=\sum_{i=1}^{m} v Z u_{i}=\sum_{i=1}^{m} \mathrm{k} v u_{i}$, and so the dimension of $V$ is at most $m$.

COROllary 2.4. Over algebraically closed fields of characteristic zero X contains no semisimple Lie algebras. 
Proof. A semisimple Lie algebra over an algebraically closed field of characteristic zero has finite-dimensional irreducible modules of arbitrarily high dimensionalities, and this would not be the case, by Theorem 2.3 , if such an algebra were in $\boldsymbol{X}$.

We recall that Max-cu $\leqslant$ Max and Max $\leqslant$ Fin- $A$. Thus by Theorem 2.1 we have that Curtis algebras are finite-dimensional and so from Theorem 1.6 we have

TheOREM 2.5. (a) Over fields of characteristic zero, Max-cu $=\mathbf{M a x}-\mathrm{pu}=\mathbf{F} \cap \mathbf{A}$.

(b) Over fields of characteristic $p, \mathbf{M a x}-\mathrm{cu}=\mathbf{F}$.

The class $\mathbf{X}$ contains a class Max-pu first mentioned in Amayo and Stewart [1] and defined as comprising Lie algebras whose universal algebras are finitely generated over a noetherian subalgebra of their centre. Thus Max-cu $\leqslant \mathrm{Max}$-pu $\leqslant$ $\mathbf{X} \cap \operatorname{Max}-\mathrm{u} \leqslant \mathbf{X} \cap \operatorname{Max}$ and $\mathbf{X} \cap \operatorname{Max} \leqslant \mathbf{X} \cap$ Fin- $\mathbf{A} \leqslant \mathbf{F}$. Now in characteristic $p, \mathbf{F}=\mathbf{M a x}-\mathrm{cu}=\mathbf{X} \cap$ Fin-A and in characteristic zero, as $\mathbf{X} \cap$ Fin- $\mathbf{A} \leqslant \mathbf{F}$, we see that $\mathbf{X} \cap$ Fin-A is closed under field extensions and quotients. Suppose that $L \in \mathbf{X} \cap$ Fin - A over a field of characteristic zero. Then to show $L$ has to be abelian we may, without loss of generality, assume that the field is algebraically closed, $L$ is finite-dimensional and for each nonzero ideal of $I$ of $L$ the quotient $L / I$ is abelian. Assume $L$ is not abelian. Then $L$ satisfies the hypothesis of Lemma 1.2. Now $L$ cannot be the 2-dimensional nonabelian Lie algebra as Example 1.5 shows; nor can $L$ be simple on account of Corollary 2.4 , and $L$ cannot be nilpotent on account of Theorem 2.2. So $L$ must be abelian after all. So we have then that $\mathbf{F}>\mathbf{M a x}-\mathrm{cu}=\mathrm{Max}-\mathrm{pu}=\mathbf{X} \cap \mathbf{M a x}=\mathbf{X} \cap$ Fin-A, over any field. In summary, we have

THEOREM 2.6. Curtis algebras are precisely those algebras whose abelian subalgebras are finite-dimensional and whose universal algebras are finitely generated modules over their centres. All such algebras are necessarily finite-dimensional.

\section{REFERENCES}

1. R. K. Amayo and I. N. Stewart, Infinite-dimensional Lie algebras, Noordhoff, Leyden, 1974.

2. __ Finitely generated Lie algebras, J. London Math. Soc. 5 (1972), 697-703.

3. R. K. Amayo, Engel Lie rings with chain conditions, Pacific J. Math. 54 (1974), 1-12.

4. C. W. Curtis, Noncommutative extensions of Hilbert rings, Proc. Amer. Math. Soc. 4 (1953), 945-955.

5. N. Jacobson, Lie algebras, Interscience, New York, 1962.

Department of Mathematics, Southern Illinois University at Carbondale, Carbondale, ILLINOIS 62901 